\title{
GLOBAL SIMULATIONS OF THE MAGNETIC FIELD EVOLUTION IN BARRED GALAXIES UNDER THE INFLUENCE OF THE COSMIC -RAY -DRIVEN DYNAMO
}

\author{
K. KULPA-DYBEє ${ }^{1}$, K. OTMIANOWSKA-MAZUR ${ }^{1}$, B. KULESZA-ŻYDZIK ${ }^{1}$, \\ M. HANASZ ${ }^{2}$, G. KOWAL ${ }^{1,3}$, D. WÓLTAŃSKI ${ }^{2}$ and K. KOWALIK ${ }^{2}$
}

\begin{abstract}
We present three-dimensional global numerical simulations of the cosmic-ray (CR) driven dynamo in barred galaxies. We study the evolution of the interstellar medium of the barred galaxy in the presence of non-axisymmetric component of the potential, i.e. the bar. The magnetohydrodynamical dynamo is driven by CRs, which are continuously supplied to the disk by supernova (SN) remnants. No magnetic field is present at the beginning of simulations but one-tenth of SN explosions is a source of a small-scale randomly oriented dipolar magnetic field. In all models we assume that $10 \%$ of $10^{51} \mathrm{erg}$ SN kinetic energy output is converted into CR energy.

To compare our results directly with the observed properties of galaxies we construct realistic maps of polarized radio emission. The main result is that the CR-driven dynamo can amplify weak magnetic fields up to a few $\mu \mathrm{G}$ within a few Gyr in barred galaxies. The obtained $e$-folding time is equal to $300 \mathrm{Myr}$ and the magnetic field reaches equipartition at time $t \sim 4.0 \mathrm{Gyr}$. Initially, completely random magnetic field evolves into large-scale structures. An even (quadrupoletype) configuration of the magnetic field with respect to the galactic plane can be observed. Additionally, the modeled magnetic field configuration resembles maps of the polarized intensity observed in barred galaxies. Polarization vectors are distributed along the bar and between spiral arms. Moreover, the drift of magnetic arms with respect to the spiral pattern in the gas density distribution is observed during the whole simulation time.
\end{abstract}

Subject headings: cosmic rays - galaxies: evolution - galaxies: magnetic fields methods: numerical

\footnotetext{
${ }^{1}$ Astronomical Observatory, Jagiellonian University, ul Orla 171, 30-244 Kraków, Poland

${ }^{2}$ Toruń Centre for Astronomy, Nicolaus Copernicus University, 87-148 Toruń/Piwnice, Poland

${ }^{3}$ Núcleo de Astrofísica Teórica, Universidade Cruzeiro do Sul, Rua Galvão Bueno 868, CEP 01506-000, São Paulo, Brazil
} 


\section{INTRODUCTION}

Bars are ubiquitous and occur in all types of disk galaxies, from early to late Hubble types. In near-infrared images about $70 \%$ of all the nearby disk galaxies are barred (Menéndez-Delmestre et al. 2007). Bars are astrophysically important not only because they are very common in disk galaxies, but they also can significantly affect the gas distribution (Sellwood \& Wilkinson 1993) as well as the magnetic field configuration. The first systematic observations of polarized radio emission from a sample of 20 barred galaxies show that their magnetic field topology is significantly more complicated than in the case of grand-design spiral galaxies (Beck et al. 2002). The strongest polarized radio emission was detected in NGC 1097 and NGC 1365 (Beck et al. 2005). The main magnetic field properties observed in the above barred galaxies can be summarized as follows. The polarized emission is the strongest in the central part of the galaxy, where the bar is present. In this region the polarized emission forms ridges coinciding with the dust lanes along the leading edges of the bar. The polarization vectors change quickly their pitch angles in the bar region whenever they are located upstream the dust lanes and this results in depolarization valley where the polarized emission almost vanishes. Near the shear shock areas the regions of vanishing polarized intensity are also observed. In the outer disk magnetic field vectors form spiral pattern with the maxima of emission along spiral gaseous arms and in interarm regions. The average total (regular and turbulent) magnetic field strength for this sample of barred galaxies is $10 \pm 3 \mu \mathrm{G}$. On the other hand, the average regular magnetic field, calculated from polarized radio emission, is equal to $2.5 \pm 0.8 \mu \mathrm{G}$. The strongest total magnetic field is detected in the central starforming regions (about $60 \mu \mathrm{G}$ in $\mathrm{NGC} \mathrm{1097)} \mathrm{and} \mathrm{in} \mathrm{the} \mathrm{radio}$ ridges along the galaxies bars $(20 \mu \mathrm{G}--30 \mu \mathrm{G}$ in NGC 1365). In the spiral arms of barred galaxies the total magnetic field is about $20 \mu \mathrm{G}$, while regular $4 \mu \mathrm{G}$. The spiral shape of the magnetic field, large pitch angles, and the observed strengths of magnetic fields indicate that the galactic dynamo works in those galaxies.

The original concept of the fast CR driven dynamo was proposed by Parker $(1992)$. Several researchers have approached this problem numerically, e.g., Hanasz et al. (2004, 2009a); Otmianowska-Mazur et al. (2009); Siejkowski et al. (2010). The CR-driven dynamo involves the following processes. CRs are continuously supplied to the galactic disk due to supernova (SN) explosions. The galactic disk stratified by gravity is unstable due to buoyancy of the magnetic field and CRs. Buoyancy effects induce the formation of magnetic loops in the frozen-in, predominantly horizontal magnetic fields. The rotation of the interstellar gas causes that magnetic field loops are twisted by the Coriolis force. Next, due to the fast magnetic reconnection small-scale magnetic loops merge to form the large-scale radial magnetic field component. The newly created magnetic field component is stretched by differential rotation, what results in amplification of the large-scale toroidal magnetic field component. 
The combined action of the above effects is sufficient to trigger the exponential growth of the large-scale magnetic field with timescales $140--250 \mathrm{Myr}$, which are comparable to the galactic rotation period.

Three-dimensional (3D) MHD numerical simulations in the shearing-box approximation have shown that the CR-driven dynamo can exponentially amplify weak magnetic fields up to few $\mu \mathrm{G}$ within few Gyr in spiral galaxies (Hanasz et al. 2004, 2009a) as well as in irregular galaxies (Siejkowski et al. 2010). What is more, some of the observed magnetic fields properties such as extended halo structures of the edge-on galaxies or the so called X-shaped structures (Soida 2005), can be explained using the CR-driven dynamo (Otmianowska-Mazur et al. 2009). The first complete global-scale 3D numerical model of the CR-driven dynamo in an axisymmetric galaxy has been demonstrated recently by Hanasz et al. (2009a). These simulations have given very interesting results and have shown that the CR-driven dynamo is one of the most promising process responsible for amplification and maintenance of galactic magnetic fields.

\section{THE INITIAL CONDITIONS AND INPUT PARAMETERS}

We investigated the evolution of the barred galaxy using the magnetized fluid approximation governed by the isothermal non-ideal MHD equations (Landau \& Lifshitz 1984). To make the set of above equations complete we include the CR transport. Following Schlickeiser \& Lerche (1985), the propagation of CR component in the interstellar medium (ISM) is described by the diffusion-advection equation

$$
\frac{\partial e_{c r}}{\partial t}+\nabla\left(e_{c r} \mathbf{v}\right)=\nabla\left(\hat{K} \nabla e_{c r}\right)-p_{c r}(\nabla \cdot \mathbf{v})+C R_{\text {source }}
$$

where $e_{c r}$ is the CR density, $p_{c r}=\left(\gamma_{c r}-1\right) e_{c r}$ is the CR pressure, $\hat{K}$ is the diffusion tensor, $\mathbf{v}$ is the velocity and $C R_{\text {source }}$ is the source term for cosmic ray energy. We assume that CR energy is added to the system by SNe explosions and that $10 \%$ of $10^{51} \mathrm{erg}$ SNe kinetic energy output is converted into CR energy, while the thermal energy is neglected. The adiabatic index $\gamma_{c r}$ for the CR fluid is set to be 14/9. Additionally, CRs are weightless, thus they only contribute to the total pressure (not to the total mass) and are included in the gas motion equation as $\nabla p_{c r}$ (Berezinski et al. 1990). Following Ryu et al. (2003), the anisotropic diffusion of the CR gas is described by diffusion tensor $\hat{K}$ as

$$
K_{i j}=K_{\perp} \delta_{i j}+\left(K_{\|}-K_{\perp}\right) n_{i} n_{j},
$$

where $K_{\|}$and $K_{\perp}$ are parallel and perpendicular (with respect to the local magnetic field direction) CR diffusion coefficients and $n_{i}=B_{i} / B$ are components of the unit vectors tangent to magnetic field lines. 
All numerical simulations were performed with the aid of the Godunov code (Kowal et al. 2009). We numerically investigated the CR-driven dynamo model in 3D in a computational box of the size $30 \mathrm{kpc} \times 30 \mathrm{kpc} \times 8 \mathrm{kpc}$, with a spatial resolution of $320 \times 320 \times 80$ grid zones in the $x, y$, and $z$ directions, respectively. The speed of sound is set to $c_{s}=5 \mathrm{~km} \mathrm{~s}^{-1}$ and the initial gas density at the galactic center $\rho_{0}$ is equal to 1.0 at $\mathrm{H} \mathrm{cm}^{-3}$. Following several detailed reviews of the theory of CR diffusion (e.g. Strong et al. 2007), the values of the CR diffusion coefficients assumed in the simulations are $K_{\|}=3 \times 10^{28} \mathrm{~cm}^{2} \mathrm{~s}^{-1}=100 \mathrm{kpc}^{2} \mathrm{Gyr}^{-1}$ and $K_{\perp}=3 \times 10^{26} \mathrm{~cm}^{2} \mathrm{~s}^{-1}=1 \mathrm{kpc}^{2} \mathrm{Gyr}^{-1}$. The resistivity coefficient is the same as in our previous work (Kulesza-Żydzik et al. 2009) and it is set to be $\eta=3 \times 10^{25} \mathrm{~cm}^{2} \mathrm{~s}^{-1}=$ $0.1 \mathrm{kpc}^{2} \mathrm{Gyr}^{-1}$, while $\beta=p_{c r} / p_{\text {gas }}$ is constant and equal to 1 in the initial condition. At the beginning of the calculation the magnetic field is not present. Following Hanasz et al. (2009a), the magnetic field is added to the galactic disk through randomly distributed SN explosions in the period of time $0.1 \mathrm{Gyr}--1.1 \mathrm{Gyr}$. During this time interval weak $10^{-5} \mu \mathrm{G}$ and dipolar magnetic field is supplied in $10 \%$ of SN remnants (Hanasz et al. 2009a). In the presented simulations the probability of a single SN event is proportional to the local gas density. After $t=1.1 \mathrm{Gyr}$ the dipolar magnetic field is not longer injected because, due to the dynamo action, its contribution starts to be insignificant. We apply the outflow boundary conditions on external domain boundaries.

Our model of the barred galaxy consists of four components: the large and massive halo, the central bulge, rotating disk of stars, and the bar. They are represented by different analytical gravitational potentials: the halo and the bulge components are described by two Plummer spheres, stellar disk is represented by the isochrone gravitational potential, while the bar is defined by the prolate spheroid (Binney \& Tremaine 1987). The bar component is introduced into the galaxy gradually in time, until it reaches its final mass $M_{b a r}$ (from $t=0.1$ Gyr to $t=0.4 \mathrm{Gyr}$ ). In order to conserve the total mass of the modeled galaxy we reduce the bulge mass, so we have $M_{b a r}(t)+M_{b}(t)=$ const during the calculations. The bar rotates with constant angular speed $\Omega_{b a r}$. All quantities which characterize the model of barred galaxy are summarized in Table 1 .

\section{Magnetic field evolution}

In Figure 1 the toroidal magnetic field component in horizontal and vertical slices is plotted. Red color represents the regions with the positive toroidal magnetic field, blue with negative, while unmagnetized regions of the volume are white. The random magnetic field component dominates initially (up to about $t \sim 1.0 \mathrm{Gyr}$ ), as it originates from randomly oriented magnetic dipoles. The ordered magnetic field is visible in the inner part of the 


\begin{tabular}{llll}
\hline Parameter & Name & Value & Units \\
\hline$M_{d}$ & Disc mass & $4.0 \times 10^{10}$ & $M_{\odot}$ \\
$a_{d}$ & Length scale of the disk & 0.6 & $\mathrm{kpc}$ \\
\hline$M_{b}$ & Bulge mass & $1.5 \times 10^{10}$ & $M_{\odot}$ \\
$a_{b}$ & Length scale of the bulge & 5.0 & $\mathrm{kpc}$ \\
\hline$M_{h}$ & Halo mass & $1.2 \times 10^{11}$ & $M_{\odot}$ \\
$a_{h}$ & Length scale of the halo & 15.0 & $\mathrm{kpc}$ \\
\hline$M_{b a r}$ & Bar mass & $1.5 \times 10^{10}$ & $M_{\odot}$ \\
$a_{b a r}$ & Length of bar major axis & 6.0 & $\mathrm{kpc}$ \\
$b_{b a r}$ & Length of bar minor axis & 3.0 & $\mathrm{kpc}$ \\
$c_{b a r}$ & Length of bar vertical axis & 2.5 & $\mathrm{kpc}$ \\
$\Omega_{b a r}$ & Bar angular velocity & 30.0 & $\mathrm{Gyr}$ \\
\hline$R_{G} B$ & Galaxy radius & 13.5 & $\mathrm{kpc}$ \\
CR & Corotation radius & 6.0 & $\mathrm{kpc}$ \\
IILR & Inner Inner Lindblad Resonance & 0.4 & $\mathrm{kpc}$ \\
OILR & Outer Inner Lindblad Resonance & 3.0 & $\mathrm{kpc}$ \\
OLR & Outer Lindblad Resonance & 8.5 & $\mathrm{kpc}$ \\
\hline
\end{tabular}

Table 1: Adopted Parameters for the Barred Galaxy Model 

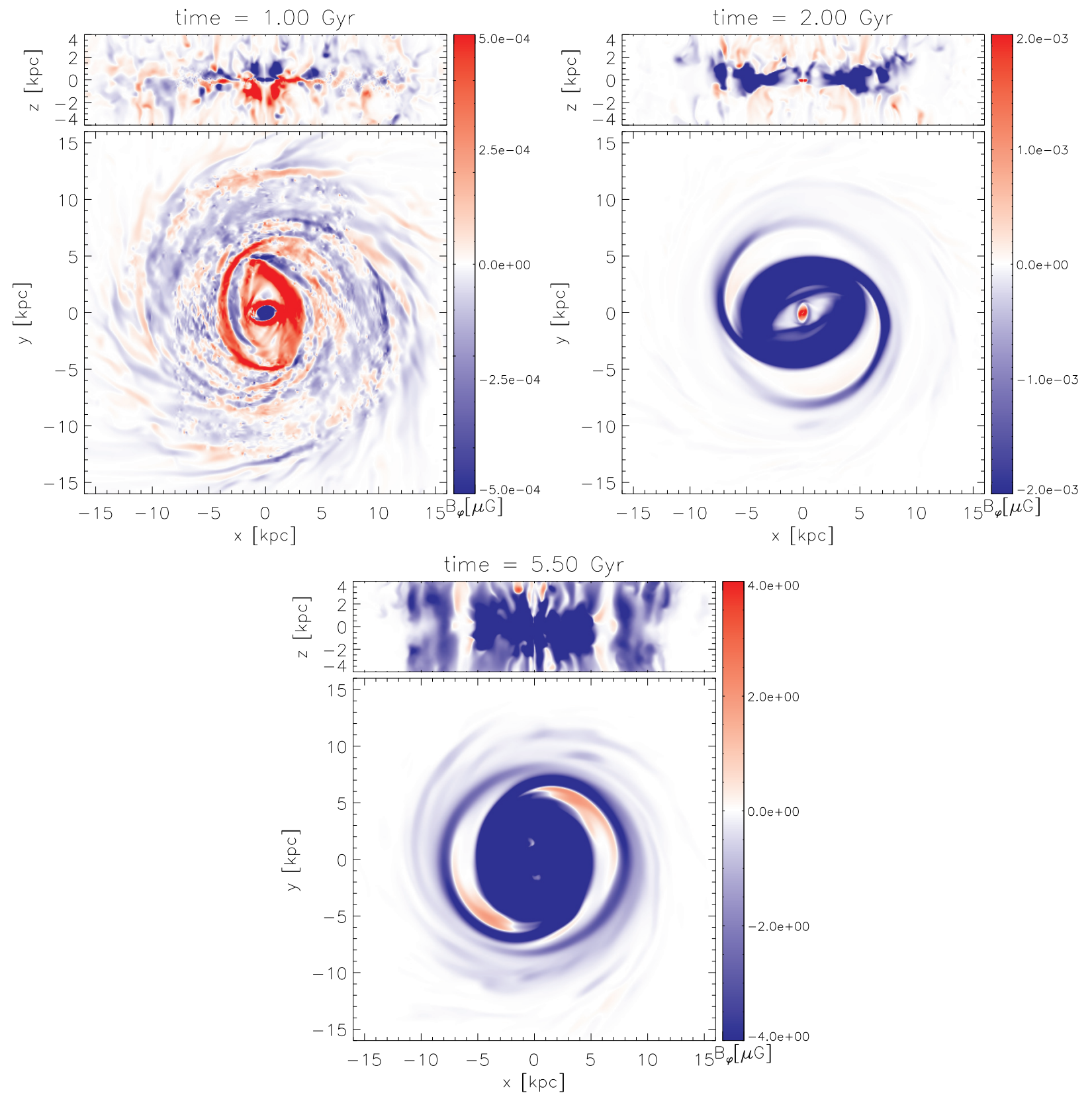

Fig. 1. - Distribution of the toroidal magnetic field in vertical and horizontal slices through the disk center for selected time steps. Red color represents the regions with the positive toroidal magnetic field, blue with negative, while unmagnetized regions of the volume are white. To enhance weaker structures of the magnetic field in the outer galactic disk (e.g., magnetic arms), the color scale in magnetic field maps is saturated. 

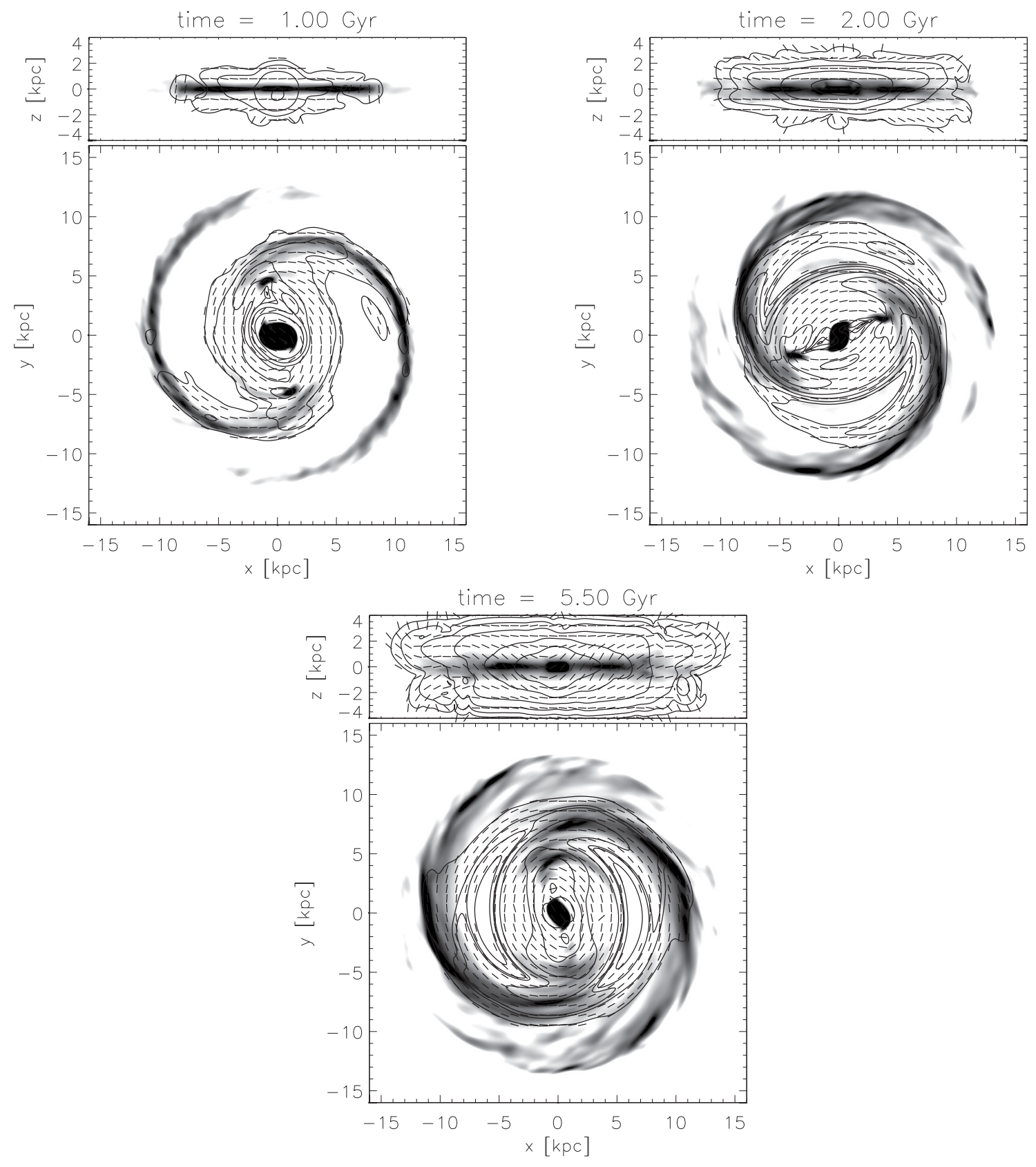

Fig. 2.- Face-on and edge-on polarization maps for selected time steps. Polarized intensity (contours) and polarization angles (dashes) are superimposed onto column density plots. 
galaxy, where it follows the gas distribution, namely the bar and dust lanes. At later epoch $(t=2.0 \mathrm{Gyr})$ the toroidal magnetic field component forms well-defined magnetic arms which can be observed till the end of the simulation.

The positive toroidal magnetic field component gradually reaches higher values, both in the bar and magnetic arms, and occupies larger volume of space in the galactic disk and halo. On the other hand, the gas motions in the bar region generate shocks which enhance the magnetic field in this area in addition to the ongoing dynamo process. Indeed, the regular magnetic field in magnetic arms at time $t=5.5 \mathrm{Gyr}$ is equal to $7.3 \mu \mathrm{G}$, while in the bar region the regular magnetic field reaches $50.1 \mu \mathrm{G}$.

Reversals of the magnetic field can be observed at time $t=2.0$ Gyr (Figure 1) in the very inner part of the bar, where the negative toroidal magnetic field component is present. However, these reversals disappear almost completely in subsequent epochs. Additionally, due to the influence of the nonaxisymmetric gravitational potential, magnetic reversals are apparent between the bar and magnetic arms at time $t=5.5$ Gyr in Figure 1 .

At the beginning of the calculation the randomly distributed toroidal magnetic field dominates what is apparent in vertical slices. Next, at time $t=1.0 \mathrm{Gyr}$ (Figure 1), the odd (dipole-type) configuration of the magnetic field with respect to the galactic plane can be observed. However, this configuration is transient and after $t=2.0$ Gyr in Figure 1 an even (quadruple-like) symmetry of the magnetic field dominates. The quadrupole-like symmetry was also obtained by Hanasz et al. (2009a) who studied the CR driven dynamo in an axisymmetric galaxies. Moreover, small reversals appear during the whole simulation time.

In Figure 2 we present the magnetic field evolution in synthetic polarization maps for the same epochs as in Figure1. The polarization maps show the distribution of the polarization angle and polarized intensity superimposed onto the column density. All face-on and edgeon polarization maps have been smoothed down to the resolution $40^{\prime \prime}$. The dark shades represent regions of the highest density. The magnetic field maxima correspond to the gas density enhancement, where the SN explosions are located, what can easily be seen at $t=1.0 \mathrm{Gyr}$ (Figure 2). At this time step, the magnetic field is present in the gaseous arms as well as in the central part of the galaxy. In the shock regions in the bar the rapid change of the magnetic field direction is apparent. Moreover, no regular magnetic field is observed in the interarm regions, however magnetic arms start to detach from gaseous spirals. At time $t=2.0 \mathrm{Gyr}$ the magnetic spiral is clearly visible between the bar and gaseous arms. The drift of magnetic arms is persistent and takes place during the whole simulation time. Its shape changes slightly as the simulation proceeds. For instance, at $t=2.0 \mathrm{Gyr}$ the magnetic spiral is well defined in the interam region, while at $t=5.5 \mathrm{Gyr}$ it is less visible because it connects 
with magnetic structures apparent in the bar region. The drift of magnetic arms into the interarm area was described in a number of papers e.g., (Kulesza-Żydzik et al. 2009, 2010), where the authors concluded that this behavior is caused by difference in the angular velocity of magnetic arms and the gaseous spirals. Namely, the magnetic arms do not corotate with gaseous spiral structure, but they follow the general gas motion in the disk, which has a

slightly lower angular velocity. However, in Kulesza-Żydzik et al. (2009, 2010) no dynamo action was included and the drift of magnetic arms into the interam region is not observed during the whole simulation time but only in the short period of calculation.

In the edge-on maps in Figure 2, the polarized synchrotron emission extends far outside the galactic plane. Near the disk plane the magnetic field is mainly parallel do the disk, while in the halo vertical magnetic field components can also be seen. The strong vertical field in the halo is probably transported by mass outflow from the galactic disk. The averaged outflow rate is equal to $1.1 M_{\odot} \mathrm{yr}^{-1}$. The most extended structures are apparent at time $t=5.5 \mathrm{Gyr}$. This configuration of the magnetic field vectors bears some resemblance to the extended magnetic halo structures of the edge-on galaxies (Krause 2009).

The CR-driven dynamo action in barred galaxies causes that the total magnetic field (left panel) and azimuthal flux (right panel) plotted in Figure 3 grow approximately exponentially. The growth of the total magnetic field lasts until the saturation level is reached at time $t=4.5 \mathrm{Gyr}$. The growth of the regular magnetic field is identified with the amplification of the azimuthal magnetic flux. The regular magnetic field grows up on an average timescale (e-folding time) equal to $300 \mathrm{Myr}$. After time $t=1.32 \mathrm{Gyr}$, the growth of the magnetic flux is clearly exponential and lasts until the equipartition is reached.

\section{Conclusion}

This work demonstrates for the first time the action of the CR driven dynamo in barred galaxies. Many observational futures of the magnetic field in barred galaxies have been reproduced.

- The polarized radio emission is strongest in the inner part of the bar and in radio ridges that approximately follow the dust lanes indicated by the enhancement of the gas density.

- In the outer part of the disk magnetic vectors form a spiral pattern with maxima of polarized intensity along spiral gaseous arms and in interarm regions. The drift of magnetic arms into the interarm area is observed during the whole simulation time. 
- The synthetic edge-on radio maps of polarized emission resemble magnetic structures observed in edge-on barred galaxies.

- The obtained average strength of the magnetic field and the maximum value of the total magnetic field in magnetic arms as well as in the bar are consistent with observational values.

- The large-scale magnetic field has a quadrupole-like symmetry with respect to the galactic plane.

The CR-driven dynamo is a powerful mechanism for amplifying magnetic fields in barred galaxies. During the whole simulation time we see the contribution driven by CRs driving turbulence, apparent through the buoyant motions. We incorporate the sub-grid physics through the resistivity of ISM. This amount of resistivity $\eta=3 \times 10^{25} \mathrm{~cm}^{2} \mathrm{~s}^{-1}$ is exactly what is needed to ensure that magnetic energy in small-scale magnetic fields and in largescale magnetic fields are equal in the CR-driven dynamo model (Hanasz et al. 2009b). The corresponding diffusive timescale is the magnetic field-ordering timescale. The timescale

$t_{\text {grow }} \sim \ln \left(B / B_{0}\right) l / v$ tells us how much the random magnetic field on scales $(l$ and $v)$ would grow in the absence of resistivity. But since, due to the resistivity, the conversion of smallscale magnetic fields to large scales is efficient, we observe the growth of the large-scale magnetic fields to the equipartition values (from the initial $B_{0}$ ), exactly in the period of 3-4 Gyr.

This work was supported by the Polish Ministry of Science and Higher Education through grants: 92/N-ASTROSIM/2008/0 and 3033/B/H03/2008/35. The computations presented here have been performed on the GALERA supercomputer in TASK Academic Computer Centre in Gdańsk.

\section{REFERENCES}

Beck, R., Shoutenkov, V., Ehle, M., Harnett, J. I., Haynes, R. F., Shukurov, A., Sokoloff, D. D. \& Thierbach, M., 2002, A\&A, 391, 83

Beck, R., Fletcher, A., Shukurov, A., Snodin, A., Sokoloff, D. D., Ehle, M., Moss, D. \& Shoutenkov, V., 2005, A\&A, 444, 739

Berezinski, V. S., Bulanov, S. V., Dogiel, V. A., Ginzburg, V. L.,Ptuskin, V. S., Astrophysics of cosmic rays, Amsterdam:North-Holland, 1990. 
Binney, S. \&Tremaine, S., 1987, Galactic Dynamics, Princeton Univ. Press, Princeton, NJ

Hanasz, M., Kowal, G., Otmianowska-Mazur, K. \& Lesch, H., 2004, ApJ, 605, L33

Hanasz, M., Wóltański, D. \& Kowalik, K., 2009, ApJ, 706, 155

Hanasz, M., Otmianowska-Mazur, K., Kowal, G. \& Lesch, H., 2009, A\&A498, 335

Kowal, G., Lazarian, A., Vishniac, E. T. \& Otmianowska-Mazur, K. 2009, ApJ, 700, 63

Krause, M., 2009, Rev. Mexicana Astron. Astrofis., 36, 25

Kulesza-Żydzik, B., Kulpa-Dybeł, K., Otmianowska-Mazur, K., Kowal, G. \& Soida, M., 2009, A\&A, 498L, 21K

Kulesza-Żydzik, B., Kulpa-Dybeł, K., Otmianowska-Mazur, K., Soida, M. \& Urbanik, M., 2010, A\&A, 522, 61

Landau, L. D. \& Lifshitz, E. M., 1984, Eloectrodynamics of Continuous Media (Oxford:Pergamon)

Menéndez-Delmestre, K., Sheth, K., Schinnerer, E., Jarrett, T. H. \& Scoville, N. Z., 2007, ApJ, 657, 790

Otmianowska-Mazur, K., Soida, M., Kulesza-Żydzik, B., Hanasz, M. \& Kowal, G., 2009, ApJ, 693, 1

Parker, E. N., 1992, ApJ, 401, 137

Ryu, D., Kim, J., Hong, S. S. \& Jones, T. W., 2003, ApJ, 589, 338

Schlickeiser, R. \& Lerche, I., 1985, A\&A, 151, 151

Sellwood, J. A. \& Wilkinson, A., 1993, Rep. Prog. Phys., 56, 173

Siejkowski, H., Soida, M., Otmianowska-Mazur, K., Hanasz, M. \& Bomans, D. J., 2010, A\&A, 510, 97

Soida, M., 2005, in The Magnetized Plasma in Galaxy Evolution, ed. K. T. Chyzy, K. Otmianowska-Mazur, M. Soida \& R.-J. Dettmar, (Kraków: Jagiellonian Univ.), 185

Strong, A. W., Moskalenko, I. V. \& Ptuskin, V. S., 2007, Annu. Rev. Nucl. Part. Sci., 57, 285 

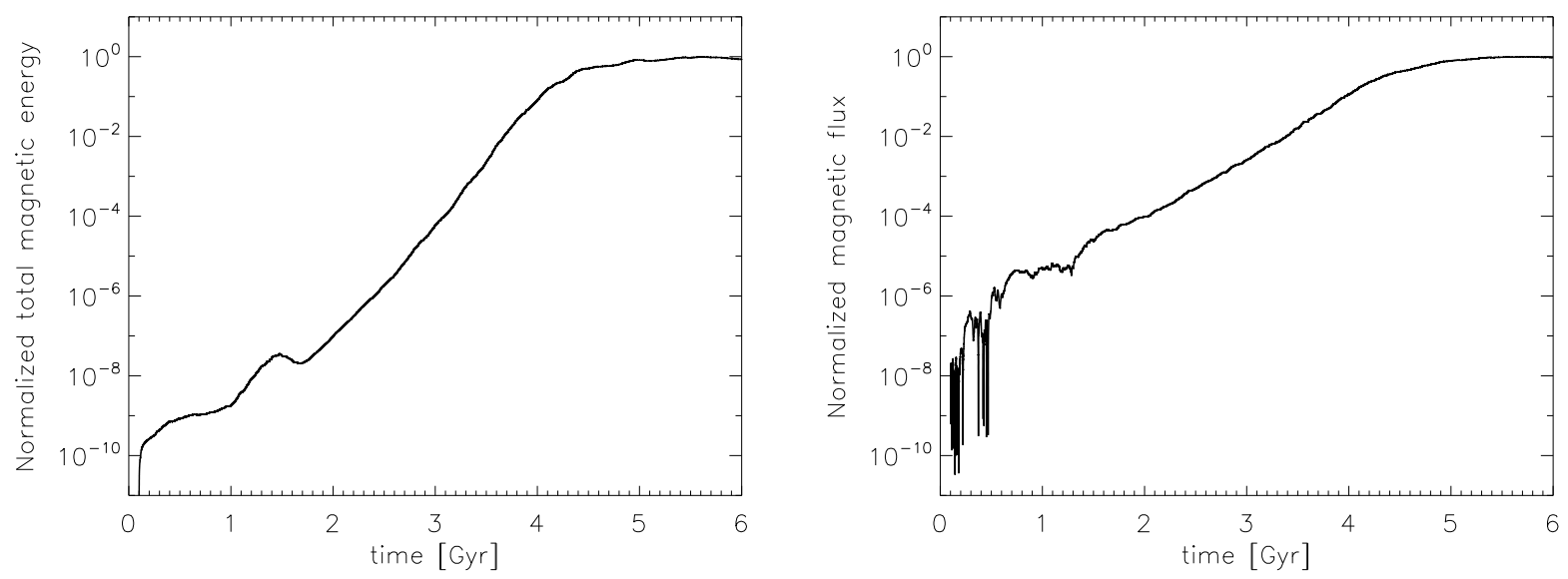

Fig. 3.- Evolution of the total magnetic energy $E_{B}$ (left panel) and the mean azimuthal flux $B_{\phi}$ (right panel) for the modelled barred galaxy. Both quantities are normalized with respect to the equipartition value. 